\title{
Les Avocats Aux Pieds Nus En Chine, Vecteurs D'accès À La Justice?
}

\section{Hélène Piquet*}

Le rôle des avocats aux pieds nus chinois, ou parajuristes, oeuvrant en monde rural est peu traité dans les études sur les réformes juridiques chinoises, en Chine et en Occident. Ce texte vise donc à situer leur contribution dans l'accès à la justice civile en Chine. Quand ont-ils émergé ? Comment travaillent-ils? Quels sont les défis et contraintes auxquels ils font face? Notre recherche montrera qu'ils jouent un rôle assez marginal et que leur avenir est très incertain.

L'approche adoptée est comparative. Il faut d'abord traiter de l'apport des parajuristes ailleurs dans le monde afin de situer celui des avocats aux pieds nus chinois. De même, l'analyse proposée est rétrospective, compte tenu du contexte politique chinois actuel. Il sera donc question, dans un premier temps, des réformes juridiques chinoises en mettant l'accent sur les mesures prises par les autorités judiciaires afin de favoriser l'accès à la justice civile. En seconde partie nous traiterons des parajuristes en général. La troisième partie consistera en une analyse du rôle des avocats chinois aux pieds nus.

The role of China's barefoot lawyers, or paralegals, working in rural areas, is scarcely addressed in studies of China's legal reforms, either in China or in the West. This study seeks to identify their contribution to access to civil justice in China. A comparative approach is used. First, the contribution of paralegals elsewhere in the world must be addressed to put China's barefoot lawyers into context. Similarly, the proposed analysis is retrospective, given the China's current political environment. Legal reforms in China will therefore be addressed first, with an emphasis on the measures taken by judicial authorities to favour access to civil justice. The second part of this study will address paralegals in general, followed by an analysis of the role of barefoot lawyers in China. When did they emerge? How do they work? What challenges and constraints do they face?

À l'heure où sévit, au nom du "maintien de la stabilité," une répression d'une ampleur sans précédent contre la société civile en Chine, ou ce qui en a tenu lieu jusqu'ici, il nous apparaît plus important que jamais de poursuivre des études sur la Chine. ${ }^{1}$ Dans cette perspective, pourquoi écrire précisément sur l'accès à la justice en Chine? Nous pensons, avec un certain recul, que parmi les réformes juridiques chinoises amorcées en 1978, la question du rôle des parajuristes chinois œuvrant en milieu rural a été

* Sinologue, Membre du Barreau; Titulaire de la Chaire de recherche du Canada Droit chinois et mondialisation UQAM, Département des Sciences Juridiques

1 Sur la répression, voir entre autres: Congressional-Executive Commission on China, 2017 Annual Report (2017), en ligne: < https://www.cecc.gov/publications/annual-reports/2017-annual-report> à la p 224; Stanley B Lubman, "Beijing's War on Rights Lawyers and Activists Continues," China Real Time Report (23 janvier 2016), en ligne:

$<$ http://blogs.wsj.com/chinarealtime/2016/01/23/beijings-war-on-rights-lawyers-and-activists-continues/tab/print/>. 
nettement moins étudiée, ce qui justifie notre entreprise. ${ }^{2}$ Quels facteurs expliquent leur essor dans le contexte du régime autoritaire chinois? Comment travaillent-ils? Jusqu'à quel point le travail des parajuristes en Chine, eu égard à leur contribution à l'accès à la justice, se compare-t-il avec celui des parajuristes ailleurs dans le monde? La question suivante fait l'objet de la présente étude: dans la mesure où dans divers pays du monde, les parajuristes sont des vecteurs d'accès à la justice, quelle a été la contribution des parajuristes chinois depuis le milieu de la décennie 1990? À la lumière des données disponibles, notre hypothèse veut que les parajuristes chinois offrent une contribution utile, mais marginale et à l'avenir incertain. Nous en explorerons les raisons plus loin.

Les organisations impliquées dans l'aide au développement et la coopération juridique ont récemment commencé à s'intéresser à d'autres canaux d'accès à la justice que les systèmes judiciaires étatiques. ${ }^{3}$ Par exemple, en 2012, le Programme des Nations Unies pour le développement (PNUD) a produit un rapport qui invitait les acteurs de la coopération internationale à prendre en compte les systèmes informels de justice. ${ }^{4}$ Cela marquait un changement de paradigme important, dans la mesure où les agences multilatérales ou les ONG amorçaient la plupart des projets de coopération en favorisant une approche institutionnelle visant la volonté de mettre en œuvre la Rule of Law (ROL). ${ }^{5}$ Les études existantes sur l'accès à la justice en Chine ne font qu'effleurer le sujet des parajuristes ou n'en traitent pas. ${ }^{6}$ Les parajuristes ont très peu retenu l'attention des juristes chinois et occidentaux, avec pour résultats un nombre infime d'études à leur sujet. ${ }^{7}$ Notre contribution ne peut pas s'appuyer sur une étude de terrain,

2 Notre perspective sera rétrospective, car il est difficile de prévoir la suite pour le système judiciaire dans son ensemble, qui connaît une situation de crise. Jerome Cohen, “A Looming Crisis for China’s Legal System,” Foreign Policy (22 février 2016), en ligne:

$<$ https://foreignpolicy.com/2016/02/22/a-looming-crisis-for-chinas-legal system/?utm_source $=$ The $\% 20$ Sinocism $\% 20$ China $\% 20$ Newsletter\&utm_campaign $=749662 \mathrm{cfa} 9$ -

Sinocism02_24_162_24_2016\&utm_medium $=$ email\&utm_term $=0 \_171 \mathrm{f} 237867-749662 \mathrm{cfa} 9$ 29590953\&mc_cid $=749662 \mathrm{cfa} 9 \& \mathrm{mc}$-eid $=92 \mathrm{a}>$.

3 Julio Faundez, "Legal Pluralism and International Development Agencies: State Building of Legal Reforms" dans Brian Z Tamanaha, Caroline Sage et Michael Woolcock, dir, Legal Pluralism and Development, New York, Cambridge University Press, 2012, 177 à la p 177.

4 UNDP, "Charting a Course for Human Rights Based Engagement" (2012), en ligne:

$<\mathrm{http}$ ://www.undp.org/content/undp/en/home/librarypage/democraticgovernance/access_to_justiceandruleoflaw/informal-justice-systems.html>

5 Fran Quigley, "Growing Political Will from the Grassroots: How Social Movement Principles Can Reverse the Dismal Legacy of Rule of Law Interventions" (2009) Colum HRLR 13.

6 Sur l'accès à la justice, à titre non exhaustif, voir Fu Hualing, "Access to Justice in China: Potential, Limits and Alternatives" (2009), en ligne: <http://ssrn.com/abstract=1474073> [Fu, "Access to Justice"]; Fu Hualing, "Access to Justice and Constitutionalism in China" dans Stéphanie Balme et Michael Dowdle, dir, Building Constitutionalism in China, New York, Palgrave Macmillan, 2009, 163; Margaret YKWoo, "Migrant Access to Civil Justice in Beijing," (2007) 4:2 Loy U Chicago IL Rev 167.

7 Ying Xing, "Barefoot Lawyers and Rural Conflicts" dans You-tien Hsing et Ching Kwan Lee dir, Reclaiming Chinese Society: The New Social Activism, New York, Routledge, 2010, 64 [Ying, "Barefoot Lawyers"]. Il s'agit de la traduction anglaise du texte paru en chinois. Ying Xing, "“Yingfa ru Xiang' "Jiejin Zhengyi”: Dui Zhongguo XiangCun ChijiaoLushi de ge an Yanjiu" ("Dissemination of Law at Village Level" and "Access to Justice": A Case Study of "Barefoot Lawyers" in China") (2007) 25:1 Zhengfa Luntan (Forum of Politics and Law) 79 [Ying, "Yin fa ru xiang"]; Tin Meuk Daisy Cheung, "Going Barefoot in the Middle Kingdom: A Preliminary Study of the Strategic Choices of 
en raison du contexte actuel. ${ }^{8}$ Toutefois, nous complèterons les études existantes en abordant le sujet des parajuristes chinois de manière comparative, afin de mettre en lumière leurs points communs, mais aussi leurs traits distinctifs par rapport aux parajuristes ailleurs dans le monde. Le terme "parajuriste" s'entend ici des personnes qui ont pour mission, qu'elles ont définie elles-mêmes ou qui leur a été confiée par divers organes étatiques ou non étatiques, de contribuer à rendre accessible le droit, compris ici comme la législation et la règlementation nationale. ${ }^{9}$ La caractéristique essentielle de ces parajuristes, qui revêt selon Cappelletti une dimension identitaire clé, réside dans "la non-appartenance aux professions légales." ${ }^{10} \mathrm{En}$ Chine, les parajuristes sont aussi appelés "avocats aux pieds nus," "barefoot lawyers" dans la doctrine de langue anglaise. Nous retiendrons cette terminologie, employée par le professeur Ying Xing dans son étude pionnière sur le sujet en Chine. ${ }^{11}$

Nous traiterons d'abord des réformes juridiques en Chine puis de quelques politiques chinoises en matière d'accès à la justice. Bien sûr, il existe un écart entre le droit légiféré et le droit vivant. Toutefois, les textes officiels permettent de dégager les positions et orientations des autorités judiciaires en matière d'accès à la justice. Cette toile de fond permettra d'aborder ensuite le sujet des parajuristes, pour en esquisser d'abord un portrait général avant de le situer dans le contexte chinois.

\section{L'ACCÈS À LA JUSTICE EN CHINE DANS SON CONTEXTE}

On peut aborder le concept d'accès à la justice selon plusieurs approches. Dans son acception étroite, l'accès à la justice est centré sur les tribunaux judiciaires et administratifs, et autres institutions d'administration de la justice, et sur le processus par lequel une personne soumet son litige à l'arbitrage. La vision large de l'accès à la justice embrasse d'autres concepts, comme le processus législatif, le contenu du droit, la légitimité des tribunaux et les modes dits alternatifs de représentation des justiciables et de résolution des différends. Enfin, l'approche intermédiaire de l'accès à la justice porte sur la résolution des conflits par des mécanismes officiels ou non officiels, mais exclut le processus législatif et le contenu du droit. ${ }^{12}$ Pour les fins de ce texte, nous combinons ces trois approches de l'accès à la justice. Elles se rejoignent en ce que les parajuristes s'insèrent plutôt dans la vision intermédiaire, mais en référence à l'acception étroite.

Non-Licensed Weiquan Lawyers in Modern China" (2013) 31:1 UCLA Pacific Basin LJ 1. Pour les fins de ce texte, nous travaillerons avec la traduction anglaise du texte de Ying Xing, sauf lorsqu'autrement précisé.

8 Chinese Human Rights Defenders, "Too Risky to Call Ourselves Defenders: CHRD Annual Report on the Situation of Human Rights Defenders in China (2015)" (2015), en ligne: <https://chrdnet.com/2016/02/too-risky-to-call-ourselvesdefenders-chrds-2015-annual-report-on-the-situation-of-human-rights-defenders-in-china/ $>$.

9 Vivek Maru, "Between Law and Society: Paralegals and the Provision of Justice Services in Sierra Leone and Worldwide" (2006) 31 Yale L J 427 aux pp 466-469 [Maru, "Between Law and Society"].

10 Riccardo Cappelletti, "Dire les droits en Afrique. Les parajuristes et les défis linguistiques" (2013), à la p 8, en ligne: $<$ https://www.academia.edu/3059957/Dire_les_droits_en_Afrique.Les_parajuristes_et_les_défis_linguistiques $>$ [Cappelletti, "Dire les droits"].

11 Ying, "Barefoot Lawyers," supra note 7.

12 Ces trois approches sont expliquées par Ghai et Cottrell. Yash Ghai et Jill Cottrell, "The Rule of Law and Access to Justice" dans Yash Ghai et Jill Cottrell, dir, Marginalized Communities and Access to Justice, New York, RoutledgeCavendish, 1 à la $\mathrm{p} 3$. 
Comme concept, l'accès à la justice apparaît dans la doctrine juridique en Amérique du Nord à partir du milieu de la décennie $1960 .{ }^{13}$ La doctrine juridique européenne apparaît peu après. ${ }^{14}$ En Chine, le concept est plus récent. Les juristes chinois ont commencé à en traiter dans la décennie 2000, notamment dans le contexte d'études portant sur la médiation judiciaire et sur le renouveau de la médiation extrajudiciaire, ou encore sur d'autres dimensions liées à la procédure civile. ${ }^{15}$

\section{A. Les réformes juridiques chinoises}

Les réformes judiciaires chinoises ont été amorcées en 1978. Pour des raisons qui lui sont propres, le Parti communiste chinois a endossé la rhétorique de la Rule of Law [ROL] comme l'un des fondements des réformes juridiques, allant jusqu'à modifier sa Constitution en 1999, pour y intégrer la notion de "Socialist Rule of Law." ${ }^{16}$ Les motivations du PCC, de même que les limites de la mise en œuvre de la ROL en Chine dépassent le cadre de cet article. Elles ont été traitées ailleurs. ${ }^{17}$ Pour nos fins, l'intérêt de cette rhétorique est qu'elle a donné lieu à plusieurs projets de coopération juridique avec la Chine axés sur le thème de la ROL. ${ }^{18}$ Cette dernière a fait l'objet d'attaques soutenues sous l'ère du précédent président de la Cour populaire suprême [CPS], Wang Shengjun. ${ }^{19}$ Toutefois, les derniers documents issus du $18^{e}$ Congrès du PCC et émanant du Comité central réhabilitent de nouveau la "Socialist Rule of Law" avec des caractéristiques chinoises. ${ }^{20}$ Cette tendance n'a pas été modifiée par le $19^{\mathrm{e}}$ Congrès.

13 Mauro Cappelletti et Bryan Garth, "Introduction," dans Mauro Cappelletti, dir, Accès à la justice et État-providence, Paris, Economica, 1984, 1 aux pp 18-20.

14 Ibid.

15 Sur le renouveau de la médiation, voir Fan Yu, Chongfen Jiejue de Lilun yu Shixian [Théorie et pratique de la résolution des conflits], Beijing, Presses de l'Université Qinghua, 2007 à la p 380; Zhu Suli "Guanyu nengdong sifa yu da tiaojie" (Sur la grande médiation) (2010) 1:153 Zhongguo Faxue 5 [Zhu, "Tiaojie"]; sur la procédure civile et l'accès à la justice, voir: Ji Shu Hao, Minshi shenqian chengxu (Civil Pretrial Procedure), coll. Access to Justice vol. 2, Xiamen, Presses de l'Université Xiamen, 2009. L'expression Access to Justice est en anglais dans l'ouvrage en chinois.

16 Chen Jianfu, Chinese Law: Context and Transformation, Leide, Martinus Nijhoff Publishers, 2015, à la p 73 [Chen, Chinese Law].

17 Pour un regard critique des juristes chinois sur le sujet, voir l'ouvrage Cai Dingjian et Wang Chenguang, dir. China's Journey Toward Rule of Law, Leide, Boston, Brill 2010. Les perspectives occidentales sur le sujet sont trop nombreuses pour être citées ici. Mentionnons les travaux pionniers de Stanley Lubman, puis de Randall Peerenboom, qui s'échelonnent sur plus d'une décennie, de même que ceux de Michael Dowdle, Jean-Pierre Cabestan et Stéphanie Balme, entre autres.

18 Les États-Unis ont été un acteur clé, avec des projets de la Ford Foundation et plusieurs autres. Le Canada a été impliqué dans un projet de formation des juges chinois, financé par l'Agence canadienne de développement international, à la fin de la décennie 1990 et au début de la décennie 2000. L’Union européenne est aussi présente, ainsi que des États membres individuels comme l'Allemagne.

19 Carl F Minzner, “China’s Turn against the Law” (2011) 9 Am J Comp L 935. [Minzner, “Against the Law”]

20 Comité central du Parti communiste chinois, "CCP Central Committee Decision concerning Some Major Questions in Comprehensively Moving Governing the Country According to the law Forward" (28 octobre 2014) China Copyright and Media, en ligne: <https://chinacopyrightandmedia.wordpress.com/2014/10/28/ccp-central-committee-decisionconcerning-some-major-questions-in-comprehensively-moving-governing-the-country-according-to-the-law-forward $>$ [Comité central, "Décision"]. 
Le système judiciaire compte au sommet une Cour populaire suprême qui, depuis peu, comporte deux divisions dans d'autres villes de Chine. ${ }^{21}$ Suivent les 31 tribunaux populaires supérieurs, répartis dans les provinces, municipalités autonomes et régions autonomes. L'échelon suivant est constitué de 400 tribunaux populaires intermédiaires, sis dans les préfectures des provinces, les municipalités relevant directement du gouvernement central, de la compétence d'une province, d'une région autonome ou d'une préfecture autonome. En milieu rural, on compte 3000 tribunaux populaires de base. Ces derniers ont le pouvoir de créer, selon les besoins, des cours ou chambres populaires de base, aujourd'hui au nombre de $17000 .{ }^{22}$

Le début des réformes en 1978 est marqué par une pénurie de juges et d'avocats. Les autorités judiciaires ont donc mis l'accent sur la formation de ceux-ci. Depuis 2001, les deux professions sont assujetties à l'obligation de réussir un concours. Aujourd'hui, la Chine compte 120000 juges. Il s'agit d'une diminution importante par rapport au chiffre de 195000 recensé par Zhu Jingwen en 2015. Cette situation s'explique par le fait que diverses réformes mises en place sous Xi Jinping ont abouti à la rétrogradation de certains juges à d'autres fonctions et ont poussé d'autres juges à embrasser une autre carrière. ${ }^{23}$ La Chine compte désormais 300000 avocats. ${ }^{24}$ En 2015, elle comptait 151092 procureurs et 12163 notaires. ${ }^{25}$

Le Parti communiste chinois [PCC] récuse la théorie de la séparation des pouvoirs. ${ }^{26} \mathrm{Il}$ en découle, entre autres, que le concept d'indépendance du pouvoir judiciaire, tel que compris dans les pays démocratiques, se transpose difficilement en Chine. ${ }^{27}$ Plus récemment, dans un document du PCC censé demeurer secret, le PCC a affirmé avec conviction son rejet de l'indépendance du pouvoir judiciaire, notamment au motif qu'il s'agirait d'un concept d'origine occidentale destiné à miner son autorite. ${ }^{28}$ Cette donne comporte d'importantes répercussions sur tout le système judiciaire chinois. Elle fait en sorte qu'il

21 Supreme People's Court, Zui Gao Renmin Fayuan Guanyu Xunhui Fating Li anjian Ruogan wenti de yijian (Supreme People's Court Provisions on Several Issues Regarding Trial of Cases in Circuit Court Divisions), China Law Translate (2015), en ligne $<\mathrm{http}: / /$ chinalawtranslate.com/spccircuitcourts/?lang=en $>$. Les traductions en anglais ne sont disponibles qu'aux abonnés. En chinois, le texte est accessible sur <http://www.court.gov.cn/fabu-xiangqing-13148.html $>$.

22 Margaret YK Woo, "The Dynamism of China's Civil Litigation System" dans Colin B Picker et Guy L Seidman, dir, The Dynamism of Civil Procedure-Global Trends and Development, New York, Springer, 2016, 141 à la p 143 [Woo, "Dynamism"]; Zhu Jingwen, Introduction to Chinese Law: Based on Data Analysis, Beijing, Presses de l'Université Renmin, Beijing, 2015, à la p 81. Ce livre est en anglais [Zhu, Introduction].

23 Pour le chiffre de 120000 juges voir "Xi: Judicial Reform Key to Rule of Law," China Daily Europe (2017), en ligne $<$ http://www.chinadaily.com.cn/m/chinalic/2017-07/14/content_30470119.htm>; pour le chiffre de 195000 juges, voir Zhu, Introduction, supra note 22 à la p 111. Sur les motifs d'insatisfaction des juges voir Carl Minzner, "Legal Reform in the Xi Jinping Era" (2015) 20 Asia Pol 4 à la p 9 [Minzner, "Legal Reform"].

24 Xinhua, "China Has 300,000 Lawyers," Xinhua (9 janvier 2017), en ligne: < http://news.xinhuanet.com/english/201701/09/c_135967814.htm>

25 Zhu, Introduction, supra note 22 à la p 111. Pour ces deux catégories, il s'agit des seuls chiffres dont nous disposons.

26 Chen, Chinese Law, supra note 16 à la p 215; Stéphanie Balme, Chine, Les visages de la justice ordinaire, Paris, Les Presses de Science Po, 2016, à la p 144.

27 Jiang Huiling, "Judicial Reform" dans Cai et Wang, dir, China's Journey toward Rule of Law, supra note 17, 199 aux pp 210-215.

28 Comité central du Bureau général du Parti communiste chinois "Communiqué on the Current State of the Ideological Sphere," ChinaFile (2013), en ligne: <https://www.chinafile.com/document-9-chinafile-translation>. L'authenticité de ce document est assurée par plusieurs sinologues. En raison des fuites dont il a fait l'objet, il est désormais bien connu. 
convient d'aborder avec nuances les cours de justice et toutes les professions juridiques. Si l'on reconnait, sur le plan formel, des institutions judiciaires en Chine, la conception de leurs rôles et leurs modes d'opération diffèrent parfois grandement des attentes des juristes occidentaux. Ainsi, en Chine, le statut du juge est peu prestigieux et on ne peut y transposer le concept de "pouvoir judiciaire." L'autorité des juges chinois n'est pas véritablement établie, ni dans le système judiciaire, ni dans la société en général. ${ }^{29}$ Depuis 1949, les avocats, dont la profession s'est développée beaucoup plus tardivement, n'ont jamais eu le monopole de la représentation des clients devant les tribunaux. ${ }^{30}$ Avec les autres professions juridiques, ils ont été largement éclipsés en Chine à partir de 1957, en raison des campagnes politiques comme la campagne anti-droitiers et la Révolution culturelle. ${ }^{31}$ Leur renaissance, à partir des années 1980 , s'effectue, comme pour les juges, dans un contexte de lourdes contraintes politiques qui fait obstacle au déploiement de leurs rôles tel que compris dans les pays démocratiques. Il faut aussi compter avec des problèmes, documentés ailleurs, de corruption, de faible professionnalisation des juges et de protectionnisme local. ${ }^{32}$

Pendant quelques années, les autorités judiciaires ont mis l'accent sur la médiation comme mode de résolution des conflits. Plusieurs problèmes en ont découlé, comme Carl Minzner le résume dans l'expression "virage contre le droit." ${ }^{\text {"33 }} \mathrm{Ce}$ retour en force de la médiation s'est inscrit dans un paradigme de populisme judiciaire décrit par Liebman et très imprégné de nationalisme anti-occidental. ${ }^{34}$ Cependant, depuis novembre 2014, les autorités chinoises ont largement rompu avec les orientations populistes du plan quinquennal 2009-2013. ${ }^{35}$ Elles reviennent notamment vers la professionnalisation de la magistrature et proclament l'État de droit socialiste comme principe directeur des réformes juridiques à venir. ${ }^{36}$ En outre, tout le système judiciaire demeure assujetti à la réalisation de l'objectif du maintien de la stabilité sociale et de l'harmonie. ${ }^{37}$

Il serait erroné de conclure que tout l'appareil judiciaire est paralysé et délaissé par la population. Selon les statistiques publiées par Zhu Jingwen en 2015, les justiciables ont eu de plus en plus recours aux tribunaux étatiques depuis 1978. Ainsi, de 1978 à 2011, le nombre d'affaires en matière civile, soumises aux tribunaux, a été multiplié par 19,8. En effet, en 1978, les tribunaux ont traité 318000 affaires alors

29 Liu Sida, "The Shape of Chinese Law" (2014) 1:2 Peking University Law Review 415 à la p 421.

$30 \quad$ Ibid à la $\mathrm{p} 429$.

31 Pitman Potter, China's Legal System, Malden, Polity Press, 2013, à la p 29; Liu, supra note 29 à la p 421.

32 Randall Peerenboom, China's Long March toward Rule of Law, Cambridge (RU), Cambridge University Press, 2002, aux pp 295-296 [Peerenboom, "Long March"]; Jiang Huiling, "Judicial Reform" dans Cai et Wang, supra note 17, 199 aux pp 203-205.

33 Minzner, "Against the Law," supra note 19.

34 Sur le populisme judiciaire, voir Benjamin Liebman, "A Return to Populist Legality? Historical Legacies and Legal Reform” dans Sebastian Heilman et Elizabeth J. Perry (dir), Mao's Invisible Hand: The Political Foundations of Adaptive Governance in China, Cambridge (MA), Harvard University Press, 2011, 165 à la p 165.

35 Congressional-Executive Commission on China, 2015 Annual Report, en ligne:

$<$ http://www.cecc.gov/publications/annual-reports/2015-annual-report>. Voir la rubrique Access to Justice, Minzner, "Legal Reform," supra note 23 à la p 4.

36 Comité central, "Décision," supra note 20. La référence à l'État de droit socialiste ne se trouve pas dans un paragraphe, mais se trouve dans la phrase qui ouvre la partie 1.

37 Woo, "Dynamism," supra note 22 à la p 150. 
qu'en 2011, ils en ont traité $6614049 .{ }^{38}$ Ces données indiquent que le système judiciaire, du moins en matière civile, revêt une certaine légitimité auprès des personnes désireuses de faire valoir leurs droits. ${ }^{39}$ Ces développements sont dus plus largement au fait qu'une partie de la population chinoise a conscience d'être titulaire de droits et possède la volonté de les défendre en recourant aux tribunaux ou à d'autres canaux..$^{40}$ Les autorités judiciaires ont pris acte de cet usage des tribunaux étatiques par la population et ont formulé, au fil du temps, diverses politiques destinées à régir le système judiciaire.

\section{B. Les orientations des politiques judiciaires en matière d'accès à la justice}

Dans la plupart des pays occidentaux, le thème de l'accès à la justice nourrit des travaux dans toute la communauté juridique, depuis les barreaux jusqu'aux tribunaux, en passant par les professeurs de droit. ${ }^{41}$ En Chine, les diverses mesures d'accès à la justice développées au fil du temps s'inscrivent dans des courants idéologiques exprimés dans des mots d'ordre, notamment comme société harmonieuse ou "hexie shehui," justice pour le peuple ou "sifa weimin," ligne de masse ou "junzhong luxian." ${ }^{22}$ L'expression chinoise pour accès à la justice est "jiejin zhengyi." L'auteur de ce texte a cherché cette expression en chinois sur Google et dans divers sites consacrés au droit en Chine mettant en ligne des articles de doctrine et des documents juridiques. ${ }^{43}$ L'expression est employée dans des articles de journaux qui commentent les réformes de la justice au Royaume-Uni ou les réformes juridiques japonaises ${ }^{44}$ Eu égard à la doctrine, l'expression renvoie à l'article de Ying Xing et à quelques rares monographies. Elle est totalement absente

38 Zhu, Introduction, supra note 22 à la p 85.

39 Pour le monde urbain, voir Mary E Gallagher, "Mobilizing the Law in China: 'Informed Disenchantment' and the Development of Legal Consciousness" (2006) 40:4 Law and Society Rev 783 à la p 794; pour le monde rural voir Fu Hualing, "Cong xiangtu shehui falü zhidu kan falü yu fazhan: chongfen de jiejue yu jingji fazhan" ("Regards sur le droit et le développement à partir de la société rurale") dans Liang Zhiping, dir, Guojia, shichang, shehui: dangdai zhongguode falü yu fazhan ( $L$ 'État, le marché, la société: le développement du droit de la Chine contemporaine) Beijing, Presses de l'Université Zhengfa, 2005, 141, particulièrement à la p 161 sur la place du droit étatique dans les revendications des paysans [Fu, "Xiangtu Shehui"]; Kevin J O'Brien et Lianjiang Li, Rightful Resistance in Rural China, New York, Cambridge University Press, 2006 à la p 6.

40 En général, Potter, supra note 31 à la p 73; Fu Hualing, "Challenging Authoritarianism through Law" dans Jean-Philippe Béjà, Fu Hualing et Eva Pils, dir, Liu Xiaobo, Charter 08 and the Challenges of Political Reform in China, Hong Kong, Hong Kong University Press, 2012, 185 à la p 191 [Fu, "Challenging Authoritarianism"].

41 À titre d'exemple, voir le rapport de Lord Woolf sur la réforme de la justice civile au Royaume-Uni. Lord Woolf, Access to Justice: Final Report (1996), en ligne:

$<$ http://webarchive.nationalarchives.gov.uk/t/http:/www.dca.gov.uk/civil/final/contents.htm>.

42 Chacun de ces mots d'ordre a inspiré nombre d'opinions et autres documents de la Cour populaire suprême. Par exemple, on retrouve "sifa weimin" dans Supreme People's Court, Guiding Opinions of the Supreme People's Court Concerning Implementing the 23 Measures for "Justice for the People" (2003), en ligne: <http://en.pkulaw.cn>. Site accessible sur abonnement seulement.

43 La recherche a été faite avec "jiejin zhengyi” en caractères chinois. Les sites visités sont: Zhongguo Minshi Chengxu Falu Wang <http://www.civilprocedurelaw.cn/>; China Court, en ligne: Zhongguo Fayuan Wang $<$ http://www.chinacourt.org/>; Zhengyiwang, en ligne: http://www.lawinnovation.com/.

44 À titre d'exemple d'un tel article, tiré du site du Fazhi Ribao (Legal Daily) sur le site Fazhi Wang, voir "Ying Guo Sifa Gongzuo Fazhan Zhanlu" ("La voie du développement pour le travail judiciaire en Angleterre"), Legal Daily (28 septembre 2012), en ligne: $<$ http://www.legaldaily.com.cn/Frontier_of_law/content/201209/28/content_3875861_4.htm>. 
des plans quinquennaux de la Cour populaire suprême et de ses divers documents, notamment les opinions et avis.

Cela dit, depuis 1978, les autorités judiciaires ont prôné et élaboré diverses mesures associées à l'accès à la justice, comme la mise sur pied d'un système d'aide juridique à partir de $1994,{ }^{45}$ des procès sur le mode "petites créances," la simplification de certaines procédures et la réduction des coûts en matière civile. ${ }^{46}$ Plusieurs de ces mesures se reflètent dans les modifications apportées en 2012 à la Loi de procédure civile de la République populaire de Chine et les mesures relatives aux petites créances ont été mises en œuvre à l'échelle du pays. ${ }^{47}$

C'est dire qu'en pratique, ces diverses mesures visent à faciliter l'accès aux tribunaux étatiques et correspondent à la définition étroite d'accès à la justice évoquée par Ghai et Cottrell. ${ }^{48}$ De même, à partir de 1985, les autorités chinoises ont régulièrement lancé des campagnes nationales d'éducation juridique ayant pour but d'inciter les justiciables chinois à recourir aux tribunaux étatiques afin de faire valoir leurs droits et résoudre les conflits. ${ }^{49} \mathrm{La}$ campagne la plus récente porte sur la diffusion de "L'État de droit socialiste." ${ }^{50}$ Toutes ces mesures s'inscrivent dans une approche des réformes juridiques, caractérisée comme institutionnelle, statocentrique, légicentrique et centrée sur les cours. ${ }^{51}$

Les autorités judiciaires ont assez tôt été conscientes de la disparité des ressources juridiques entre la ville et les campagnes chinoises. Tout d'abord, un retour sur la notion de monde rural s'impose. Selon Fu Yulin, il s'agit des districts et des cantons au sein desquels existent des villes et des bourgs, ou des villages. ${ }^{52}$ Woo note que les avocats, concentrés dans les villes, sont en nombre insuffisant pour répondre aux besoins de services juridiques de la population rurale. ${ }^{53}$

Au fil du temps, les autorités judiciaires ont mis sur pied diverses institutions, inégalement réparties, destinées à fournir des services juridiques dans le monde rural. En raison de leur rôle important, nous privilégierons le système des travailleurs juridiques de base [TJB]. Ces derniers répondent à la définition de parajuriste, car ils sont, à l'instar des avocats aux pieds nus, des non-professionnels du droit. Tout comme les avocats aux pieds nus, ils sont issus des communautés qu'ils desservent et fournissent des

45 Fu, “Access to Justice," supra note 6 à la p 17.

46 Supreme People's Court, Zuigao Renmin Fayuan guanyu jin yibu zuohao sifa gengminlimin gongzuo de yijian (Supreme People's Court Opinion on Furthering Judicial Efforts to Facilitate and Benefit the People) (2014), para 8 en

ligne: $<$ http://chinalawtranslate.com/courtfaciliation/?lang=en $>$, note: Sur le site ChinaLaw Translate son titre est traduit par "SPC Opinion on Access to Justice" [CPS, Opinion 2014].

47 Kristie Thomas, "Dynamism in China's Civil Procedure Law: Civil Justice with Chinese Characteristics" dans Picker et Seidman, supra note 22, 119 aux pp 120-121.

48 Ghai et Cottrell, supra note 12 à la p 3.

49 Zhang Ming Xin, "Dui dangdai Zhongguo Pufa Huodong de Fansi" ("Réflexions sur les campagnes d'éducation juridique en Chine contemporaine") dans Xu Zhang Run, dir, Pufa Yundong (Les mouvements d'éducation juridique), Beijing, Presses de l’Université Qinghua, 2011, 177 à la p 177.

50 Comité central, "Décision," supra note 20, à la partie 5, au para 1.

51 Fu, "Access to Justice," supra note 6 à la p 2. L'expression anglaise employée par Fu est "court-centric."

52 Fu Yulin, "Dispute Resolution and China's Grassroots Legal Services" dans Margaret YK Woo et Mary E Gallagher, dir, Chinese Justice. Civil Dispute Resolution in Contemporary China, New York, Cambridge University Press, 2011, 314 à la 314 [Fu, "Dispute Resolution"].

53 Margaret YK Woo, "Law's Location in China’s Countryside" (2012) 29: 2 Wis Intl L J 416 à la p 425 [Woo, "Law’s Location"]. 
services juridiques abordables. ${ }^{54}$ La première différence fondamentale entre les TJB et les avocats aux pieds nus réside dans le fait que depuis le début des réformes, ils sont soutenus par les autorités judiciaires chinoises. Les TJB sont encadrés par des politiques judiciaires officielles du ministère de la Justice, dont ils relèvent. ${ }^{55}$ En outre, divers gouvernements locaux en Chine ont édicté, au fil du temps, des mesures et des politiques qui leur sont favorables. ${ }^{56}$ La seconde différence tient au fait que les TJB sont très bien intégrés dans les institutions locales et travaillent en étroite collaboration avec les gouvernements locaux et la police. ${ }^{57}$ En échange du soutien du ministère de la Justice dont ils bénéficient, ils consacrent une partie de leur temps aux dossiers qui leur sont confiés par les gouvernements locaux en matière civile et administrative.$^{58}$ En outre, les TJB veillent à maintenir de bonnes relations avec les juges et avec le personnel judiciaire.

Les TJB sont apparus dans le paysage juridique chinois dans les années 1980 sous d'autres noms. ${ }^{59} \mathrm{Ils}$ œuvrent dans les Bureaux de services juridiques [BSJ] uniquement en milieu rural. Les BSJ doivent s'autofinancer et, en conséquence, ils facturent leurs services aux justiciables. ${ }^{60}$ Si le nombre de TJB a connu une augmentation dans la décennie 1990, leur nombre a diminué par la suite. Ils étaient 121904 en l'an 2000. En 2011, leur nombre est passé à 73 000. Zhu Jingwen estime qu'il existe une moyenne de 5,42 TJB pour chaque tranche de 100000 habitants. ${ }^{61}$ Selon des données récentes, le nombre de TJB est stable. ${ }^{62}$ Le ministère de la Justice leur a conféré une vaste compétence. Les TJB sont habilités à traiter de dossiers dans presque tous les domaines à l'exception du droit pénal et de ceux qui sont couverts par les notaires. ${ }^{63}$ Au milieu de la décennie 1990, les TJB avaient plus de dossiers à traiter que les avocats. ${ }^{64}$

Fin 2017, le ministère de la Justice a promulgué un décret (ling) visant à institutionnaliser le système des TJB en milieu rural. Ce décret constitue une consécration officielle importante. Le ministère de la Justice est un acteur majeur sur le plan institutionnel. Sans être le plus puissant, ses divers décrets et ordonnances touchent à de multiples volets de la justice chinoise. En termes de hiérarchie des normes du droit chinois, un décret ne revêt pas le même prestige qu'une loi édictée par l'Assemblée populaire

54 Fu, "Dispute Resolution," supra note 52 à la p 328; Chen Xuan, Wo Guo Jiceng Falu Fuwu Gongzuo zhe de Xianzhuang yu Fazhan dui ce yanjiu (Recherches sur le développement et la situation réelle des travailleurs juridiques de base de mon pays), Beijing, Presses de l’Université Zhengfa, 2015 à la p 157.

55 Chen, Chinese Law, supra note 16, à la p 222.

56 Michael Ng et Xuanming Pan, "Nonprofessional Access to Justice in Rural China: a History of Atypical Legal Development and Legal Service Provision" (2017) 17:3 China Review 59 aux pp 75-76, en ligne, $<$ https://muse.jhu.edu/article/676364>.

57 Fu Hualing, "Away from Grassroots? The Irony of Rural Legal Service” (2012) à la p 11, en ligne: $<$ http://ssrn.com/abstract=2063115> [Fu, "Away from Grassroots"].

$58 \quad \mathrm{Ng}$ et Pan, supra note 56 à la p 72.

59 Ibid, historique complet des TJB.

$60 \mathrm{Fu}$, "Dispute Resolution," supra note 52 à la p 321.

61 Zhu, Introduction, supra note 22 à la p 122.

62 Sifabu (ministère de la Justice), "QuanGuo jiceng falu fuwu gongzuozhe 73000 zhiye zhun rumen kanjiang tigao" ("The Raising of Qualifications for the Whole Country's 73,000 Basic Level Legal Workers"), China.Com (4 janvier 2018), en ligne: $<$ http://news.china.com/socialgd/10000169/20180104/31914711.html >. Le portail est en plusieurs langues. Nous avons travaillé avec la version chinoise.

$63 \mathrm{Ng}$ et Pan, supra note 56 à la p 72.

64 Ibid à la $\mathrm{p} 73$. 
nationale. Toutefois, le décret en question a la même force contraignante. L'objet du décret est de systématiser et de rehausser les exigences d'accès au statut de TJB. Il donne suite à un vieux projet. ${ }^{65}$

L'agence de presse Xinhua a interviewé un responsable du ministère sur le décret. Le ministère a affirmé que les TJB jouaient un rôle irremplaçable comme pourvoyeurs de services juridiques dans les zones rurales des provinces économiquement sous-développées. Il s'agit d'un exemple d'institution juridique "avec caractéristiques chinoises." 66 Le décret identifie expressément les TJB, mais passe entièrement sous silence les avocats aux pieds nus.

Dans plusieurs pays, les régimes d'aide juridique constituent une mesure classique pour favoriser l'accès à la justice, perçu comme l'accès au système judiciaire et à l'arbitrage. En Chine, ce régime, formalisé par un règlement du Conseil des affaires d'État en 2003, est financé par les gouvernements locaux. ${ }^{67}$ En vertu de l'article 13 du règlement, ces derniers peuvent déterminer le seuil d'admissibilité à l'aide juridique en fonction des conditions locales. Toutefois, comme c'est souvent le cas, il ne répond pas, tant s'en faut, à tous les besoins, car il ne couvre que les plus pauvres d'entre les pauvres. Ceci a pour effet d'exclure une partie importante de la population, en ville comme en milieu rural. ${ }^{68}$ C'est dans ce contexte marqué par la pénurie de services et de ressources juridiques que sont apparus les avocats aux pieds nus, ou parajuristes du monde rural chinois.

\section{LES PARAJURISTES: UNE DIVERSITÉ DE RÔLES ET DE PRATIQUES À TRAVERS LE MONDE}

L'intérêt récent pour l'accès à la justice et les parajuristes dans le monde de la coopération est lié à l'approche dite "bottom-up," destinée à remplacer l'approche "top-down." Elle se révèle, à l'usage, insatisfaisante aux yeux de plusieurs acteurs de la coopération juridique lorsqu'il s'agit d'ouvrir la justice aux groupes défavorisés. ${ }^{69}$ Cette approche "bottom-up" s'inscrit dans une nouvelle compréhension du terme "développement," et surtout, dans une critique du paradigme de la Rule of Law (ROL) qui a dominé les projets de coopération juridique pendant deux décennies. ${ }^{70}$ L'une de ces approches "bottom-up" est celle du Legal Empowerment of the Poor [LEP], ou capacitation juridique des pauvres. ${ }^{71}$

65 Chen, Chinese Law, supra note 16, à la p 222.

66 Sifa Bu (ministère de la Justice), Zhonghua renmin gongheguo Sifa bu ling (Décret du ministère de la Justice de République populaire de Chine) di 138 hao (no 138), Jiceng falu fuwu gongzuozhe guanli banfa (Méthode de gestion des travailleurs juridiques de base), Legal Daily (Quotidien juridique) (25 décembre 2017), en ligne: <http:// www.legaldaily.com.cn/zt/content/2018-01/02/content_7437974.htm?node=90470>.

67 Guowu yuan (Conseil des affaires d'État), Falu xiezhu Tiaoli (Règlement sur l'aide juridique) (2003), en ligne: $<$ http://en.pkulaw.cn>. Depuis, plusieurs provinces et autres entités administratives locales ont édicté une règlementation sur l'aide juridique.

68 Fu, “Access to Justice," supra note 6 à la p 23.

69 Benjamin Van Rooij, "Bringing Justice to the Poor, Bottom-up Legal Development Cooperation" (2012) 4 Hague Journal on the Rule of Law 286 à la p 304.

$70 \quad$ Ibid aux pp 296-297.

71 Il existe plusieurs définitions du LEP et leur traitement dépasse le cadre de cet article. Chaque institution multilatérale, de même que les auteurs proposent des définitions dont le contenu et les orientations politiques varient. 
Le recours aux parajuristes, dans plusieurs pays d'Asie, d'Afrique et d'Amérique latine, est une des applications pratiques de l'approche "bottom up." ${ }^{, 2}$ Nous dresserons un portrait général des parajuristes avant de traiter du cas chinois. Cette démarche permettra de mieux situer, le cas échéant, les apports et les limites des parajuristes chinois.

\section{A. Quelques caractéristiques des parajuristes}

Il n'existe pas de définition universellement reconnue des parajuristes. Toutefois, celle dite du Statut des parajuristes, développée à Bamako, établit un certain consensus, car elle a été élaborée et ratifiée par plusieurs ONG travaillant en Afrique. ${ }^{73}$ Aux termes de l'article premier: "Est parajuriste, la personne (homme ou femme) connaissant les réalités sociologiques de son milieu d'intervention, jouissant d'une notoriété certaine et qui contribue à rendre le droit accessible aux populations." ${ }^{.74} \mathrm{~L}$ 'article second contient l'élément clé qui distingue le parajuriste: "Le ou la parajuriste n'est pas un ou une professionnel(le) du droit.". 75

Les parajuristes travaillent dans des contextes politiques variés. Ils ne sont pas l'apanage des pays démocratiques, car certains évoluent dans des États marqués par la répression civile et politique. ${ }^{76}$ Dans ce dernier cas, outre la Chine, signalons que des parajuristes ont travaillé en Afrique du Sud dès la décennie 1960, sous l'apartheid, et aux Philippines sous la dictature de Ferdinand Marcos. ${ }^{77}$ On retrouve des parajuristes en grand nombre en Afrique, mais aussi en Asie (Inde, Indonésie, Philippines) et en Amérique latine.

Vivek Maru a regroupé plusieurs traits communs aux parajuristes, et les lignes qui suivent sont fondées sur ses propos. ${ }^{78}$ Tout d'abord, les parajuristes travaillent ou comme généralistes, ou selon une approche de spécialisation des questions juridiques qu'ils traitent. Ils sont originaires de la communauté à laquelle ils offrent leurs services. ${ }^{79}$ Selon le cas, ils agissent à titre bénévole ou sont des employés rémunérés. ${ }^{80} \mathrm{Ils}$ œuvrent le plus souvent en milieu rural, tel qu'il ressort des projets étudiés par Golub et Maru.

72 Ibid à la p 293. Voir aussi Stephen Golub, "Beyond Rule of Law Orthodoxy, The Legal Empowerment Alternative" (2003) aux pp 33-34, en ligne: Carnegie Endowment <http://carnegieendowment.org/files/wp41.pdf>.

73 Cappelletti, "Dire les droits," supra note 10 à la p 6.

74 Statut du parajuriste, Article premier, Cadre national de pilotage du curriculum de la formation du parajuriste (1998), en ligne: $<$ http://www.cnpcpmali.org/pages/documentation.html .

75 Ibid art 2.

76 Maru, "Between Law and Society," supra note 9 à la p 466. Maru donne l'exemple du Zimbabwe.

77 Pour l'Afrique du Sud, voir: Jackie Dugard et Katherine Drage, "To Whom the People Take Their Issues? The Contribution of Community-Based Paralegals to Access to Justice in South Africa," World Bank Justice and Development Working Paper series, World Bank (2013) à la p 5, en ligne: <http://wwwwds.worldbank.org/external/default/WDSContentServer/WDSP/IB/2013/07/10/000333037_20130710110554/Rendered/ PDF/793390NWPJ0D0T00PUBLIC00Box0377373B.pdf>; pour les Philippines, voir Jennifer Franco, Hector Soliman et Maria Roda Cisnero, "Community Based Paralegalism in the Philippines: From Social Movements to Democratization," World Bank Justice and Development Working Paper series, World Bank (2014) aux pp 4-5, en ligne: <http://wwwwds.worldbank.org/external/default/WDSContentServer/WDSP/IB/2014/03/12/000333037_20140312112758/Rendered/ PDF/860000NWP0Comm00Box382162B00PUBLIC0.pdf $>$.

78 Maru, "Between Law and Society," supra note 9 aux pp 466-470.

79 Ibid à la $\mathrm{p} 470$.

80 Vivek Maru, "Access to Justice and Legal Empowerment: A Review of World Bank Practice" (2010) 2 Hague Journal on the Rule of Law 259 à la p 268 [Maru, "Access to Justice"]. 
Ce sont des gens ordinaires qui travaillent directement avec les pauvres ou autres groupes désavantagés pour traiter de problèmes d'accès à la justice et de droits de la personne. Ils recherchent des solutions concrètes aux problèmes d'accès à la justice des personnes qu'ils aident. En Afrique, les parajuristes évoluent souvent dans un contexte de pluralisme juridique où diverses normes (d'origine coutumière, religieuse) coexistent avec celles du droit édicté par l'État. ${ }^{81}$ Ils utilisent les unes et les autres, selon les affaires à traiter, parfois en les combinant. ${ }^{82}$ La médiation extrajudiciaire constitue un des outils des parajuristes en matière de résolution des conflits, mais non le seul. ${ }^{83}$

Les relations des parajuristes avec le système judiciaire étatique et les professions juridiques varient d'un pays à l'autre. Van Rooij fait état d'un bilan plutôt positif des programmes de parajuristes financés par des projets de coopération dans divers pays. ${ }^{84}$ Les parajuristes, quel que soit le modèle dans lequel ils opèrent, répondent à des besoins en matière de services juridiques. Toutefois, pour bien comprendre l'apport des parajuristes, il faut tenir compte de plusieurs facteurs. Ainsi, le territoire couvert par rapport à la superficie du pays et à la répartition de la population peut tempérer le caractère positif de certains bilans. ${ }^{85}$

Les programmes de parajuristes financés par des institutions internationales connaissent plusieurs limites. Par exemple, il s'avère difficile de former comme parajuriste des personnes ne possédant aucun bagage juridique. ${ }^{86}$ De même, le retrait des donateurs à la fin des projets met en péril la survie des programmes des parajuristes. ${ }^{87}$ Or, le cas chinois diffère de manière notable en ce que les parajuristes, ou avocats aux pieds nus, ont émergé sans le soutien d'organisations internationales et par conséquent, n'ont pas été placés dans une situation de dépendance à un financement extérieur. Ils font face à d'autres défis liés au contexte du pays.

\section{B. Les parajuristes chinois, travailleurs invisibles}

Nous avons effectué une recherche sur Baidu et ensuite sur Google avec les caractères chinois pour "chijiao lushi." Le nombre d'articles émanant de sources journalistiques officielles chinoises est inférieur à 10 pour la période 2007-2017. ${ }^{88}$ Nous éviterons à dessein l'emploi de l'expression "weiquan," qui veut dire défense des droits, pour parler des avocats aux pieds nus ou parajuristes. ${ }^{89}$ En Chine, cette dernière expression est d'abord associée, tel qu'il ressort des travaux d'Eva Pils et de Fu Hualing, à une minorité

81 C'est ce qu'Étienne Le Roy appelle le dédoublement des modes de résolution des conflits, en vertu duquel coexistent les modes formels étatiques avec les modes informels, dont le caractère légal varie. Étienne Le Roy, Les Africains et l'Institution de la Justice: Entre mimétismes et métissages, Paris, Dalloz, 2004, à la p vii.

82 Maru, "Between Law and Society," supra note 9 à la p 470.

83 Maru, “Access to Justice," supra note 80 à la p 272.

84 Van Rooij, supra note 69 aux pp 304-306.

85 Ibid à la p 307.

86 Ibid à la p 306.

87 Ibid à la p 307.

88 Ce fait peut être interprété comme illustrant le peu d'intérêt que les parajuristes suscitent.

89 Nous omettons délibérément le cas bien documenté de Chen Guangcheng, avocat aux pieds nus. Son parcours, et notamment le fait d'avoir reçu l'aide de l'ambassade américaine pour sortir de Chine, le distingue des autres avocats aux pieds nus. Voir son livre, Chen Guangcheng, L'avocat aux pieds nus, Paris, Globe, 2015. 
d'avocats en titre voués à la défense des droits de la personne, pris dans une conception large..$^{90}$ Bien sûr, on pourrait dire que les avocats aux pieds nus qui œuvrent à la défense des droits en général font littéralement du "weiquan" et peuvent être légitimement désignés ainsi. ${ }^{91}$ Toutefois, nous ne souscrivons pas à cette approche. Les "parajuristes" et "avocats aux pieds nus" se distinguent des avocats du weiquan et des travailleurs juridiques de base. ${ }^{92}$ Enfin, il faut aussi préciser, en référence au texte original en langue chinoise de Ying Xing, qu'il emploie l'expression "avocats aux pieds nus" ou "chijiao lushi" comme équivalent de "représentant des citoyens" ou"gongmin daili."

Selon Ying Xing, les avocats aux pieds nus ont commencé à travailler dans la décennie 1990. Ils répondent tout à fait à la définition du parajuriste en ce qu'ils ne sont pas des professionnels du droit. Leur essor s'explique par les orientations des réformes juridiques alors cultivées par les dirigeants et les autorités judiciaires de Chine. Avec précaution, durant la décennie 1990, les autorités locales ont laissé diverses initiatives "bottom up" voir le jour, dans un contexte où le langage des droits à défendre s'est répandu dans la société chinoise. Ces initiatives s'appuyaient sur la formulation vague de la ROL qui leur a permis d'expérimenter et de prendre des risques en matière de réformes juridiques. ${ }^{94}$ Les parajuristes chinois ont donc vu le jour et œuvré dans ce climat politique particulier, qui a aussi fait une place variable et ténue à l'essor des organisations sociales et à la société civile chinoise en général. ${ }^{95}$ Néanmoins, ces développements se situent dans un contexte de régime autoritaire qui définit et détermine l'espace conféré à ces acteurs ainsi que les limites imposées à leurs actions. ${ }^{96}$

Le travail des avocats aux pieds nus repose sur une assise juridique certaine, soit l'article 58 de la Loi de procédure civile de la République populaire de Chine. ${ }^{97}$ Cet article a été modifié entre la période où

90 Eva Pils, China's Human Rights Lawyers: Advocacy and Resistance, New York, Routledge, 2015. Voir aussi Fu Hualing et Roy Cullen, "Weiquan (Rights Protection) Lawyering in an Authoritarian State: Building a Culture of Public-Interest Lawyering" (2008) 59 China Journal 111.

91 C'est le choix de Cheung, supra note 7, qui assimile les deux.

92 Fu Hualing emploie en effet l'expression avocats aux pieds nus (barefoot lawyers) pour désigner les travailleurs juridiques de base. Fu, "Access to Justice," supra note 6 à la p 10. Nous verrons en quoi les deux groupes sont différents. Notre choix terminologique qui distingue les deux est confirmé par le réseau de presse officiel chinois Renmin Wang ou People.cn. Voir l'article: "Liu xun Laoren zixue falu bian 'chijiao lushi' sike wuran ershi nian" ("Un vieil homme se forme tout seul au droit, devient avocat aux pieds nus et combat désespérément la pollution depuis 20 ans"), Renmin wang (People.cn) (24 juillet 2017), en ligne: <http://legal.people.com.cn/n1/2017/0724/c42510-29424405.html> ["Liu xun Laoren"].

93 Ying, "Yin fa ru xiang," supra note 7 à la p 80. La traduction anglaise reprend cette terminologie. Les "gongmin daili" sont surtout actifs dans le delta de la rivière des Perles et se concentrent sur les droits des travailleurs migrants employés dans les usines.

94 Carl Minzner, "Legal Reform," supra note 23, aux pp 7-8. Sur la société civile chinoise dans le contexte de l'autoritarisme, voir Jessica C Teets, Civil Society under Authoritarianism: The China Model, Cambridge, MA, Cambridge University Press, 2014.

95 Carl Minzner, "China After the Reform Era" (2015) 26:3 Journal of Democracy 129 à la p 133, en ligne: $<$ http://www.journalofdemocracy.org/authoreditor/carl-minzner> [Minzner, "China After"].

96 Rappelons que Fu, "Challenging Authoritarianism," supra note 40, emploie l'expression autoritarisme pour qualifier le régime chinois.

97 Comité permanent de l'Assemblée populaire nationale, Civil Procedure Law of the People's Republic of China, en ligne: $<$ http://en.pkulaw.cn>. Ce site contient les modifications apportées aux lois et il est donc possible de consulter des versions plus anciennes et des versions plus récentes de la législation. 
Ying Xing (début des années 2000) a effectué ses recherches et celles où Cheung a interviewé des avocats aux pieds nus (2014). Cheung voit une différence de taille entre les deux textes, mais nous ne souscrivons pas à son interprétation. ${ }^{98}$ Ainsi, la version opérationnelle de l'article 58 dans la décennie 2000 prévoyait que des parties pouvaient nommer une ou deux personnes comme leur agent ad litem afin de les représenter. Cette personne pouvait être, selon le cas, un avocat, un proche parent des parties, une personne recommandée par un groupe social pertinent ou une unité à laquelle les parties sont liées ou tout autre citoyen approuvé par la Cour. ${ }^{99}$ La Loi de procédure civile de la République populaire de Chine fut modifiée en 2012. Aux termes du nouvel article 58, des personnes qui ne sont pas des avocats en titre peuvent représenter des amis ou parents dans des procédures judiciaires, à condition que la communauté ou l'organisation sociale pertinente ait recommandé leur implication. ${ }^{100}$ Cheung interprète la première version comme donnant le droit à une personne approuvée par la Cour d'agir comme avocat aux pieds nus, et les amendements comme ajoutant l'exigence de l'approbation par d'autres entités. ${ }^{101}$ Or, selon notre interprétation, le fait de pouvoir agir avec l'accord de la Cour ne constitue pas la seule voie ouverte aux avocats aux pieds nus pour représenter un justiciable. Il s'agit d'une possibilité, l'autre consistant à être une personne recommandée par le groupe social pertinent ou l'unité à laquelle elle est rattachée. L'approbation par la Cour ne figure plus dans la nouvelle version. En revanche, l'exigence d'être une personne approuvée par la communauté ou l'organisation sociale pertinente demeure. Le fait que la possibilité d'agir avec l'accord de la Cour disparaisse constitue-t-il un problème? Faute de données sur la question, nous ne pouvons pas répondre. Est-il difficile pour les avocats aux pieds nus de se faire recommander par leur communauté ou organisation sociale? Sans données précises, nous ne pouvons que supposer que cette exigence, même si elle n'est pas nouvelle, pourrait créer des difficultés, car donner l'approbation peut comporter un risque pour la communauté ou l'organisation sociale. Dans ce cas de figure, la disparition de la possibilité de faire valider l'avocat aux pieds nus par la Cour représente une perte. Par contre, rien ne dit que les cours, dans un contexte politique incertain, seraient plus enclines à donner leur accord que les organisations sociales pertinentes ou la communauté.

Contrairement aux avocats en titre, les avocats aux pieds nus ne peuvent pas être rémunérés pour leur travail. ${ }^{102}$ Ils doivent donc exercer d'autres activités afin de subvenir à leurs besoins. ${ }^{103}$ Fait essentiel, les avocats aux pieds nus n'ont aucune existence officielle, car ils ne sont pas enregistrés auprès du ministère de la Justice ni d'aucune autre institution étatique. Leur clientèle se constitue essentiellement par le bouche à oreilles et parfois grâce à la publicité favorable des médias chinois, puisque les tribunaux leur donnent souvent gain de cause. ${ }^{104}$ Au chapitre des facteurs de succès, Ying Xing estime déterminante la capacité des avocats aux pieds nus de s'insérer dans les logiques relationnelles de la société rurale, dominées par

98 Cheung, supra note 7 à la p 21.

99 Comité permanent de l'Assemblée populaire nationale, Civil Procedure Law of the People's Republic of China, Beijing, Foreign Languages Press, 2000. L'auteur a conservé cette vieille version dans cet ouvrage qui constitue une traduction officielle de la CPL.

100 Ibid.

101 Cheung, supra 7 note à la p 21.

102 Ying, "Barefoot Lawyers," supra note 7 à la p 65; Cheung, supra note 7 à la p 7.

103 Cheung, supra note 7 à la p 12.

104 Ying, "Barefoot Lawyers," supra note 7 à la p 75. Ce constat vaut pour la période étudiée par Ying Xing car depuis, les avocats aux pieds nus sont pratiquement absents des médias chinois. 
des règles non juridiquement contraignantes, mais ressenties comme telles par les justiciables. Il s'agit d'une relation fondée sur les sentiments "renqing" plutôt que sur le profit. ${ }^{105}$ De même, le fait qu'ils soient issus des communautés dont sont originaires les plaignants constitue un autre élément clé de leur succès, car les relations de proximité dominent la société rurale chinoise. ${ }^{106}$ Or, cet élément de proximité avec les populations desservies est également considéré comme très positif et important pour les parajuristes en général. ${ }^{107}$ En effet, comme le souligne Riccardo Cappelletti, ce lien avec le "local" renforce la légitimité des actions des parajuristes. ${ }^{108}$

Dans un texte récent, Liu Sida souligne pour sa part que les justiciables du monde rural chinois ne font pas forcément la différence entre les avocats en titre, les parajuristes et les travailleurs juridiques de base. ${ }^{109}$ Ils sont en quête de solutions à leurs problèmes et retiendront les personnes qu' ils estiment à même de les aider. Il est particulièrement important qu'ils perçoivent que la personne choisie possède de bonnes relations avec le tribunal et est en mesure d'influer sur l'issue du litige. Pour Liu, il s'agit là d'un élément central beaucoup plus important que le fait de détenir ou non un permis d'exercice de la profession d'avocat. Les avocats aux pieds nus interviewés par Ying Xing et Cheung sont spécialisés dans un domaine d'intervention. ${ }^{110}$ Nous ne pouvons toutefois pas affirmer que ces constats s'appliquent à tous les avocats aux pieds nus du monde rural en Chine.

Ces derniers travaillent généralement seuls en ce qu'ils ne sont pas intégrés ou en réseau avec une organisation sociale chinoise, contrairement aux "gongmin daili" spécialisés en droit du travail dans le delta de la rivière des Perles (dans la province du Guangdong, "l'atelier du monde"). ${ }^{111}$ Le degré de collaboration des avocats aux pieds nus avec les avocats en titre dans le monde rural en subit les conséquences. Cette collaboration constitue une donne fréquente pour les "gongmin daili" du delta de la rivière des Perles, mais pas pour les avocats aux pieds nus du monde rural. Ces derniers répondent aux besoins de services juridiques découlant du manque d'avocats dans le monde rural. ${ }^{112} \mathrm{D}$ 'autre part, Ying Xing fait état de critiques hostiles par les avocats en titre à l'endroit des avocats aux pieds nus, qu'ils taxent de "hei lushi"ou avocats illégaux. ${ }^{113}$ L'on peut déduire de cet état de choses que s'il existe des relations de collaboration entre des avocats aux pieds nus du milieu rural et des avocats en titre, elles ne constituent pas la règle. Pour autant, l'absence d'intégration dans une organisation sociale ne signifie pas une absence de réseau. Ying Xing mentionne également l'effet de démultiplication découlant de la

105 Ying, "Barefoot Lawyers," supra note 7 à la p 74. Sur la place des renqing en milieu rural, voir aussi Ellen Oxfeld, Drink Water but Remember the Source: Moral Discourse in a Chinese Village, Berkeley, University of California Press, 2010 à la $\mathrm{p} 47$.

106 Ibid.

107 Maru, "Between Law and Society," supra note 9 à la p 470.

108 Cappelletti, "Dire les droits,"supra note 10 à la p 10.

109 Liu, "Shape of Chinese Law," supra note 29 à la p 430.

110 Cheung, supra note 7 à la 12; Ying, "Barefoot Lawyers," supra note 7 à la p 76.

111 Pan Xuanming, "Toward Legal Empowerment: An Empirical Account of Grassroots Labor Organizations in the Pearl River Delta of China," Chinese University of Hong Kong (2015) à la p 12, en ligne:

$<$ http://www.law.cuhk.edu.hk/en/research/crj/download/papers/Pan-2012-

Toward_Legal_Empowerment_CRJ_working_paper.pdf $\$$. L'expression "organisations sociales" désigne les ONG, avec les adaptations nécessaires dans le contexte chinois.

112 Cheung, supra note 7 à la p 19.

113 Ying, "Barefoot Lawyers," supra note 7 à la p 80. 
présence d'un avocat aux pieds nus dans un lieu donné. ${ }^{114}$ Il se constitue graduellement par la suite un réseau informel d'avocats aux pieds nus, d'abord à l'échelle du lieu concerné. Ces réseaux grandissent et se développent ensuite à l'échelle de la province. Ils revêtent même parfois une dimension interprovinciale. ${ }^{115}$ Cette situation découle entre autres de la nécessité, pour les avocats aux pieds nus, de se protéger. ${ }^{116}$

Dans le contexte chinois et d'après les données dont nous disposons, les avocats aux pieds nus ne sont pas perçus par les communautés qu'ils desservent comme une force sociale susceptible de provoquer des changements. ${ }^{117}$ Cette situation est très différente de celle qui caractérise la place des parajuristes au Mali. Ainsi, dans le cas du Mali, le parajuridisme est "un projet de société."118 Il revêt une dimension collective, car les parajuristes sont choisis parmi la population du village afin de "suivre une formation qui leur permettra d'informer et d'orienter la population du village." "19 On discerne également une dimension collective dans le cas des parajuristes aux Philippines, aussi appelés "auxiliaires juridiques." Ces derniers ont été formés par des groupes d'intérêt public en droit. ${ }^{120} \mathrm{Au}$ contraire, dans le cas chinois, il ressort des données présentées par Ying Xing et par Cheung que les avocats aux pieds nus ont décidé eux-mêmes de devenir parajuristes, suite à diverses expériences. Leur choix est purement individuel, sans consultation avec des instances de leur communauté d'appartenance. Le cas de Luo Li Quan, qui travaille dans un district de la municipalité autonome de Chongqing, est tout à fait typique à cet égard. C'est son contact personnel avec la pollution de l'eau, causant la mort de poissons en grande quantité, qui l'a conduit à devenir avocat aux pieds nus. ${ }^{121}$

Les parajuristes sont animés par une volonté très claire de travailler "dans le système" (tizhinei) et non hors de celui-ci. ${ }^{122}$ Tout en étant en marge du système judiciaire et au fait de ses maux décrits ailleurs, ils recherchent le contact avec celui-ci. Ce dernier est à leurs yeux un forum légitime de résolution des conflits.

L'un des apports majeurs des parajuristes, selon Maru, réside dans leur capacité à travailler dans un contexte de pluralisme juridique compris ici comme la présence d'un répertoire normatif incluant d'autres normes que celles édictées par l'État, notamment le droit coutumier. ${ }^{123}$ Le pluralisme juridique pose la question des sources du droit, plus particulièrement "la mise en évidence de ses manifestations non

\footnotetext{
114 Ibid à la p 71.

115 Ibid à la p 78.

116 Ibid.

117 Il s'agit d'une déduction de l'auteur au regard des sources accessibles.

118 Leandro Varison, "Des droits au cœur du "village." Le travail des parajuristes au Mali” (2010) Cahiers d'anthropologie du droit 89 à la p 90.

119 Ibid à la p 89.

120 Carl Cesar Rebuta, Ronald A Gregorio et Yasmin O. Hatta, Des avocats aux pieds nus: Défendre les droits des communautés aux Philippines, Foncier et développement (2012) à la p 4, en ligne:

$<$ http://www.foncier-developpement.fr/publication/des-avocats-aux-pieds-nus-defendre-les-droits-des-communautesaux-philippines/>.

121 “Liu xun Laoren,"supra note 92.

122 Cheung, supra note 7 à la p 16. Ying ne fait aucune affirmation à cet effet, mais nous pouvons déduire cela de son étude de terrain.

123 Maru, "Between Law and Society," supra note 9 à la p 470.
} 
étatiques." 124 Or, la théorie des sources qui prévaut en Chine reflète une vision moniste, qui exclut la reconnaissance de toute forme de pluralisme juridique. ${ }^{125}$

Cette capacité de travailler dans un contexte de pluralisme juridique, présentée comme un atout par Maru pour les parajuristes, est-elle une donne pertinente dans le cas des avocats aux pieds nus en Chine? Afin de répondre à la question, il faut d'abord traiter du pluralisme en Chine selon quatre perspectives. Dans un premier temps, considérons ce qu'en dit la doctrine. Dans un deuxième temps, tâchons de déterminer, à la lumière des sources disponibles, si les juges chinois en milieu rural sont ouverts à d'autres arguments que ceux fondés sur le droit étatique. En troisième lieu, examinons la nature des arguments invoqués par les avocats aux pieds nus. Quatrièmement, prenons en compte la considération, par les justiciables, de normes autres que celles du droit édicté par l'État.

Sur le premier point, des chercheurs et des juges affirment depuis plusieurs années l'existence d'autres sources de normativité que le droit étatique dans la société rurale chinoise. ${ }^{126}$ Un des ouvrages clés sur le sujet demeure celui de Fei Xiaotong. ${ }^{127}$ Le juge You Tiancheng a également beaucoup écrit sur le sujet. ${ }^{128}$ Le professeur Zhu Suli, ancien doyen de la Faculté de droit de l'Université de Beijing, a consacré plusieurs ouvrages et articles à la question. ${ }^{129}$ Nous pouvons donc tenir pour acquis que la société rurale puise dans un vaste répertoire normatif au sein duquel la norme de droit étatique ne constitue qu'un élément, telle que la pratique ancienne d'exiger que la famille du mari paye une dot à la famille de l'épouse. ${ }^{130}$ Le mariage reste par ailleurs assujetti à l'ensemble de la législation applicable. Toutefois, cette autre

124 Hugues Moutouch, "Pluralisme juridique" dans Denis Alland et Stéphane Rials dir, Dictionnaire de la culture juridique, Paris, PUF, 2003, à la p 1160.

125 C'est ce qui ressort du chapitre consacré aux sources du droit par Jianfu Chen dans son ouvrage. Jianfu Chen, Chinese Law, supra note 16 à la p 253. Dans ce chapitre, l'existence de la normativité du monde rural est totalement passée sous silence. Chen présente la conception chinoise des sources du droit qui, bien que non identifiée comme telle, est une approche moniste. Il n'est fait aucune mention d'une situation de pluralisme juridique, même non reconnu, en Chine.

126 Yu Zhong, Xiangtu Zhongguo de Sifa Tujing (Les canaux de la justice de la Chine rurale), $2^{\mathrm{e}}$ édition, Beijing, Law Press, 2013; You Tiancheng, Xiangtu Shehui zhong de Minjian Fa (Le droit vivant de la société rurale), Beijing, Law Press, 2005 [You, Le droit vivant]; Sun Liping, "Les armes faibles des forts. L'usage des normes sociales informelles dans l'exercice du pouvoir" dans Isabelle Thireau et Huan Linshan dir, Disputes au village chinois. Formes du juste et recomposition locale des espaces normatifs, Paris, Éditions de la Maison des Sciences de l'Homme, 2001, 249 aux pp 281 ss. Sun étudie la place de l'adage "heqing heli hefa" (en accord avec les sentiments, en accord avec la raison, en accord avec le droit) dans la résolution de certains conflits entre les villageois et les cadres locaux du Parti. La plupart des contributions de cet ouvrage traitent de la normativité qui régit le monde rural et de la place variable du droit étatique dans celle-ci.

127 Fei Xiaotong, Xiangtu Zhongguo (La Chine rurale), Shanghai, Presses du groupe Le siècle de Shanghai, 2007. Il s'agit d'une réédition, la première édition remontant à 1984.

128 You Tiancheng, "Xiangtu Shehui Zhong de Guojia yu Minjian Fa" ("Le droit étatique et le droit vivant de la société rurale") (2001) 25:7 Yunnan Daxue Renwen Shehui Kexue Xue Bao (Yunnan University's Journal of Humanities and Social Sciences) 81; You Le droit vivant, supra note 124.

129 Zhu Suli, Song Fa Xia Xiang: Zhongguo jiceng sifa zhidu yanjiu (Faire descendre le droit vers les campagnes. Recherche sur le système judiciaire de base), Beijing, Presses de l'Université de Beijing, 2000. L'ouvrage a été réédité en 2011.

130 "Curbing the Trend of Rising Bride Price," China Daily (23 février 2017), en ligne:

$<$ http://www.womenofchina.cn/womenofchina/html1/news/china/1702/4684-1.htm>. Cet exemple constitue un rare cas documenté où les autorités législatives locales prennent acte d'une pratique et tentent, non de l'abolir, mais de la règlementer. 
normativité n'est que partiellement prise en compte par les autorités judiciaires chinoises, qui le plus souvent continuent de privilégier une approche statocentrique et institutionnelle des réformes juridiques. D'ailleurs, le juge You Tiancheng a déploré le fait que tout le processus législatif écarte totalement cette autre normativité. Il a émis la suggestion que le législateur chinois incorpore certains éléments de la normativité du monde rural dans le droit étatique. ${ }^{131}$ Fait intéressant, en 2015, d'autres juges chinois ont abondé dans son sens. ${ }^{132}$ Ces propos, tenus à 10 ans d'intervalle, témoignent du refus persistant des autorités judiciaires chinoises de reconnaître l'existence du pluralisme juridique de facto et de jure en Chine. Certains auteurs et juges émettent donc des opinions en dissonance au discours officiel quant à la manière dont sont présentées les sources du droit en Chine. Ils font état d'autres réalités sur le terrain. En d'autres termes, nous constatons ici l'écart entre "le droit des livres" et "le droit vivant."

Concernant le deuxième point, il existe peu d'études sur le recours par les juges des tribunaux de base en milieu rural à des normes autres que celles issues du droit étatique pour résoudre un litige, le cas échéant. Fu Hualing, dans son étude sur le sujet, affirme que le virage du milieu de la décennie 2000 vers la médiation a entraîné une revalorisation de la coutume locale par certains juges qui ont commencé à l'utiliser. ${ }^{133}$ Toutefois, la plupart des juges évitent de recourir officiellement à la coutume locale et vont plutôt chercher à appliquer la norme étatique qui s'en rapproche le plus, le cas échéant. ${ }^{134}$ Sur cette base, il est impossible, faute d'informations suffisantes, de dégager un portrait d'ensemble valable pour tout le territoire chinois qui permette de poser les juges du monde rural comme des acteurs de facto du pluralisme juridique. Ces limites sont très importantes lorsqu'il s'agit de situer le rôle des avocats aux pieds nus en Chine par rapport aux parajuristes ailleurs dans le monde. En effet, si les juges chinois sont très réceptifs à des arguments fondés sur la coutume locale, cela a pour effet d'élargir la palette d'outils à la disposition des avocats aux pieds nus qui comparaissent devant eux. Dans le cas contraire, le recours à des instruments autres que la norme de droit édictée par l'État ne constitue pas une option pour les avocats aux pieds nus. Malgré le peu d'informations dont nous disposons quant au troisième point, il est possible d'esquisser des éléments de réponse. Il ressort de l'étude de Ying Xing que les avocats aux pieds nus privilégient l'usage de la norme de droit étatique, à laquelle ils vouent un grand respect. ${ }^{135}$ Les avocats aux pieds nus interviewés par Cheung usent d'un répertoire normatif plus varié, qui inclut parfois des arguments faisant appel à la morale. ${ }^{136}$ Cheung ne donnant pas de précisions sur la teneur de ces arguments, il est impossible d'affirmer qu'il s'agit de la coutume locale. Quoi qu'il en soit, le recours aux arguments extrajuridiques

131 You, Le droit vivant, supra note 124 aux pp 181-182.

132 Li Yu Jiao, Duan Li Tong et Liu Huan Zi, "Ruhe Zengqiang Nongmin Fazhi guannian” (2015) (Comment augmenter la compréhension de l'État de droit chez les paysans), en ligne: <http://xtzy.hebeicourt.gov.cn/public/detail.php?id=846>. Ce document est disponible sur le site de la Haute Cour populaire de la province du Hebei et constitue un exemple d'une prise de position publique par des juges chinois sur des sujets liés à l'administration de la justice [Li, Duan et Liu, "Comment augmenter"].

$133 \mathrm{Fu}$, “Access to Justice," supra note 6 aux pp 34-35.

134 Ibid à la $\mathrm{p} 37$.

135 Ying, "Barefoot Lawyers," supra note 7 à la p 79.

136 Cheung, supra note 7 à la p 17. 
ne rallie pas tous les avocats aux pieds nus et la norme de droit étatique demeure un outil très important dans leur travail. ${ }^{137}$

Qu'en est-il maintenant du comportement des justiciables du monde rural eu égard à l'usage de normes autres que la règle de droit édictée par l'État? Cheung ne traite pas du tout de la question. ${ }^{138}$ Il nous reste celle de Ying Xing, qui jette un éclairage précis sur le sujet. Il démontre que les justiciables du monde rural peuvent vouloir s'approprier, à leurs propres fins, la norme de droit étatique. ${ }^{139}$ En d'autres termes, loin de bouder celle-ci, ils la recherchent. En 2010, Fu Hualing a aussi constaté que les justiciables du monde rural n'ont pas recours au droit étatique. Toutefois, il ne voit pas dans ce fait un rejet délibéré de ce dernier. Selon $\mathrm{Fu}$, les raisons tiennent d'abord à des problèmes d'accès à la justice. ${ }^{140}$

La taille et la géographie du territoire chinois rendent vraisemblable l'hypothèse que dans des villages très reculés et difficiles d'accès, les populations règlent d'abord leurs conflits en fonction de règles locales autres que celles du droit étatique. Par ailleurs, les villages sont affectés, à divers degrés, par les migrations des jeunes qui partent travailler en ville. Il en découle un va-et-vient d'idées et de nouvelles manières de voir susceptibles, à terme, de modifier les traditions villageoises. Toute tradition peut évoluer. ${ }^{141} \mathrm{C}$ 'est vrai des normes de droit coutumier du monde rural qui peuvent être transformées au contact du droit étatique. ${ }^{142} \mathrm{Il}$ se crée donc une relation dynamique entre les deux, engendrée par les avocats aux pieds nus. ${ }^{143}$

Ying Xing a effectué ses recherches de terrain avant le grand virage en faveur de la médiation, judiciaire et extrajudiciaire, décrété par les autorités judiciaires chinoises dès 2007. Il traite uniquement des recours devant les tribunaux étatiques. L'étude de Cheung ne mentionne pas non plus la médiation dans le travail des avocats aux pieds nus qu'il a interviewés. Il est difficile de généraliser à partir de deux études qualitatives espacées dans le temps, mais il est loisible de supposer que la médiation, si elle est présente, ne constitue pas une composante centrale du travail des avocats aux pieds nus.

La question qui se pose maintenant est celle de l'avenir des avocats aux pieds nus en Chine. À cette fin, il est utile de faire un rappel d'autres mécanismes de résolution des conflits en Chine rurale. Les pétitions reposent sur une autre logique que les recours devant les tribunaux et constituent un sujet en soi, qui dépasse le cadre de ce texte. ${ }^{144}$ Les pétitionnaires qui usent du système ont de plus en plus de mal à y accéder et sont régulièrement en butte à des représailles. ${ }^{145} \mathrm{Ce}$ canal officiel de résolution des conflits demeure très sollicité et a traité, à certaines époques, plus d'affaires que les tribunaux en matière civile. ${ }^{146}$ Les justiciables du monde rural peuvent aussi, comme évoqué plus tôt, se tourner vers les travailleurs juridiques de base. Ces derniers, comme les avocats aux pieds nus, sont des non-professionnels du droit.

137 Ibid à la $\mathrm{p} 18$. Il convient de distinguer les arguments présentés aux tribunaux des moyens utilisés par les avocats aux pieds nus. Pour nos fins, seuls les fondements des arguments soumis au tribunal nous intéressent.

Il ne s'agit pas d'un reproche, mais d'un constat, ses recherches visant d'autres objets.

139 Ying, "Barefoot Lawyers," supra note 7 à la p 73.

$140 \mathrm{Fu}$, "Xiangtu Shehui," supra note 39 aux pp 156-160.

141 Ying, "Barefoot Lawyers," supra note 7 à la p 70.

142 Ibid à la $\mathrm{p} 71$.

143 Ibid à la $\mathrm{p} 79$.

144 Carl F Minzner, "Xinfang: An Alternative to Formal Legal Institutions" (2996) 42: 1 Stan J Intl L 103.

145 Congressional-Executive Commission on China, supra note 1 à la p 271.

146 Thomas, supra note 47 aux pp 121-122. 
Toutefois, depuis le début des réformes, avec le soutien du ministère de la Justice, ils ont conquis une place très importante dans plusieurs zones rurales défavorisées des provinces économiquement sousdéveloppées, et leur volume d'activité est considérable. Un autre facteur important de cette appréciation réside dans les objectifs des justiciables en quête de justice. Ils sont intimement liés à leur vision de ce qui, dans un contexte donné, constitue une solution juste. ${ }^{147} \mathrm{Si}$ la simple reconnaissance du bien-fondé de leur grief compte plus qu'une indemnisation financière obtenue suite à un procès devant le système judiciaire étatique, alors le système des pétitions ou encore la médiation pratiquée par les TJB répond mieux à leurs besoins. ${ }^{148}$ Cette appréciation suppose au préalable que les justiciables d'une région donnée ont le choix entre ces diverses ressources juridiques. Il faut aussi compter avec les décisions des justiciables lorsqu'ils disposent de plus d'une ressource juridique. Vers qui se tournent-ils? Le contexte politique qui règne en Chine incite plus que jamais à la prudence. Les propos de Liu sur les critères motivant les choix des justiciables nous conduisent à supposer que les justiciables préfèreront recourir aux TJB, bien intégrés dans les institutions locales, avec lesquelles ils ont de bonnes relations, plutôt qu'aux avocats aux pieds nus dont les liens avec les institutions locales sont souvent ténus, voire antagonistes.

Toutefois, si les avocats aux pieds nus constituaient les seules ressources juridiques dans les zones rurales des provinces économiquement sous-développées, nous pourrions affirmer qu'ils seraient appelés à jouer un rôle crucial en matière d'accès à la justice pour les justiciables de ces régions, à l'instar des parajuristes dans certains pays d'Afrique et ailleurs dans le monde. Dans l'état actuel des choses, compte tenu de la forte présence des TJB, appelée à augmenter si l'on se fie au décret du ministère de la Justice, ils risquent plutôt de devenir redondants, s'ils ne le sont pas déjà.

Ce premier scénario reste cependant sujet à l'augmentation effective du nombre de TJB. Or, il est trop tôt pour prédire l'effet du décret du ministère de la Justice. Il pourrait aussi avoir pour effet de rendre plus difficile l'accès au statut de TJB. Si cela se produit, le plan du ministère serait compromis. Dans cette perspective, les avocats aux pieds nus conserveraient, en apparence, un plus grand espace pour se déployer. Or, le contexte politique, particulièrement depuis l'arrivée au pouvoir de l'actuel président Xi Jinping, se traduit par une répression farouche de toute la société civile chinoise. ${ }^{149}$ Cette situation va-telle, à terme, dissuader les avocats aux pieds nus de continuer leur travail? Si, pour les avocats aux pieds nus, le prix à payer pour continuer à travailler est de devenir des agents du "maintien de la stabilité," au nom de laquelle ils sont par ailleurs réprimés, certains pourraient décider d'imiter les juges et de gagner leur vie autrement. ${ }^{150}$ Pour autant, les avocats aux pieds nus, comme les avocats du weiquan, sont habitués à affronter divers registres d'adversité. Les derniers rapports sur la situation en Chine font état de l'activité continue d'avocats du weiquan en dépit de la persécution dont ils font l'objet. ${ }^{151}$ Cela témoigne d'une très grande force de conviction. Or, en Chine, être avocat aux pieds nus constitue une vocation, mue par une grande soif de justice et d'aide aux justiciables. Ces traits les rapprochent des avocats weiquan. Aussi

147 Nous renvoyons aux divers travaux sur la normativité en milieu rural cités aux notes 126 à 129 ainsi qu'aux travaux d'Oxfeld cités à la note 105 .

148 Sur le rôle des pétitions en la matière, voir: Isabelle Thireau et Hua Linshan, Les ruses de la démocratie: Protester en Chine, Paris, Seuil, 2010 à la p 407.

149 Congressional-Executive Commission on China, supra note 1 aux pp 268-272.

150 Minzner, "Legal Reform," supra note 23 aux pp 8-9. Minzner explique les divers motifs d'insatisfaction des juges sous $\mathrm{Xi}$ Jinping et l'exode de la profession qui en découle.

151 Congressional-Executive Commission on China, supra note 1 aux pp 268-272 
peut-on penser que certains avocats aux pieds nus choisissent de persister, même s'il est impossible de le vérifier.

L'article de juillet 2017 sur Luo Li Quan paru dans le très officiel réseau Renminwang suggère une autre possibilité: certains avocats aux pieds nus pourraient être tentés de briguer le statut de TJB. ${ }^{152} \mathrm{Ce}$ rare article sur le sujet des avocats aux pieds nus, reproduit sur plusieurs réseaux journalistiques de province, présente Luo sous un jour très favorable. ${ }^{153}$ Toutefois, l'article se termine sur la volonté exprimée de Luo de se qualifier en vue de devenir TJB. Faut-il voir là un message des autorités invitant les avocats aux pieds nus à rejoindre les structures officielles?

$* * *$

Les avocats aux pieds nus peuvent, selon le cas, constituer de très précieux vecteurs d'accès à la justice en milieu rural dans plusieurs pays, notamment en Afrique. L'état des lieux les concernant est marqué par la diversité dans leurs niveaux de formation, dans leurs modes d'opération et dans leurs relations avec les autres acteurs de la justice. En Chine, Ying Xing fait ressortir que l'apport des avocats aux pieds nus consiste à rendre accessible la norme de droit étatique aux justiciables du monde rural. ${ }^{154} \mathrm{Ce}$ faisant, les justiciables sont intégrés dans l'ordre judiciaire de l'État grâce aux avocats aux pieds nus. Cette contribution est qualitativement importante pour les personnes qui désireraient résoudre leurs conflits par l'entremise des tribunaux et ne seraient pas à même de le faire sans les avocats aux pieds nus. Le nombre d'études existantes sur les avocats aux pieds nus ne permet pas de déterminer, en termes quantitatifs, leur impact réel sur la justice en milieu rural. Nous ignorons leur nombre. En outre, compte tenu des autres ressources juridiques, comme les travailleurs juridiques de base et l'accès au système des pétitions, l'importance des avocats aux pieds nus diminue. Cela est directement lié à la question, plus large, de l'importance des tribunaux comme forums de résolution des conflits en Chine. Une véritable mise en perspective suppose de disposer de données fiables et récentes sur tous les canaux de justice accessibles et utilisés par la population en milieu urbain et rural à l'échelle du pays. Faute de telles données, la réflexion demeure forcément qualitative.

Dans d'autres pays, l'essor des parajuristes est lié en partie à l'existence de financement extérieur et de projets de coopération. En Chine, les avocats aux pieds nus se sont constitué tels en réaction à des donnes purement locales, sans référence aux concepts qui ont cours dans le monde de la coopération internationale, comme la capacitation juridique des pauvres. Toutefois, ils n'en sont pas si éloignés. Rétrospectivement, on peut affirmer que les avocats aux pieds nus ont été inspirés et portés par le "langage des droits" de la décennie 1990, à l'instar des autres acteurs de la société civile et d'une partie de la population chinoise. ${ }^{155}$ Pour l'heure, qui s'éternise, force est de constater que ce langage des droits, s'il

152 "Liu xun Laoren," supra note 92. Le réseau Renminwang est la plate forme du PCC qui s'exprime dans le Renmin Ribao (Quotidien quotidien du peuple).

153 Selon nous, il n'est pas anodin que cet article paraisse quelques mois avant le décret du ministère de la Justice visant les qualifications des travailleurs juridiques de base.

154 Ibid à la p 80.

155 Fu, "Challenging Authoritarianism," supra note 40 aux pp 189-192. Le titre du livre Xia Yong, dir, Zou xiang quanli de shidai: Zhongguo Gongmin quanli fazhan yanjiu (En marche vers l'ère des droits: recherche sur le développement des droits des citoyens chinois), Beijing, Presses de l'Université Zhengfa, 2000, reflète bien l'esprit qui régnait alors chez les juristes et une partie des acteurs de la société civile. 
habite toujours les esprits d'une partie de la population et des acteurs de la justice en Chine, ne peut plus résonner beaucoup. 